Research Paper

\title{
Ki67 Proliferation Index as a Histopathological Predictive and Prognostic Parameter of Oral Mucosal Melanoma in Patients without Distant Metastases
}

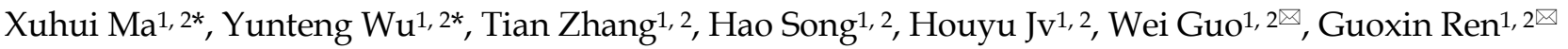 \\ 1. Department of Oral and Maxillofacial-Head and Neck Oncology, Ninth People's Hospital, Shanghai JiaoTong University School of Medicine, Shanghai, \\ 200011, China; \\ 2. Shanghai Key Laboratory of Stomatology \& Shanghai Research Institute of Stomatology; National Clinical Research Center of Stomatology. \\ * XH Ma and YT Wu contributed equally to this article. \\ $\triangle$ Corresponding authors: Address: 639 Zhi Zao Ju Road, Shanghai, China, 200011, Tel: +86-21-50398070; fax: +86-21-63136856; e-mail: guoweicn@yahoo.com \\ (W Guo) and renguoxincn@hotmail.com (GX Ren). \\ (c) Ivyspring International Publisher. This is an open access article distributed under the terms of the Creative Commons Attribution (CC BY-NC) license \\ (https://creativecommons.org/licenses/by-nc/4.0/). See http://ivyspring.com/terms for full terms and conditions.
}

Received: 2017.05.09; Accepted: 2017.07.23; Published: 2017.10.17

\begin{abstract}
Background: To investigate the relationship between clinical and histopathological characteristics and overall survival of patients with oral mucosal melanoma (OMM) without distal metastasis in order to provide predictive prognostic information of OMM.

Methods: Ki67 expression was assessed by immunohistochemistry in 123 patients with OMM without distant metastases. The associations between Ki67 expression and clinical features and overall survival (OS) of patients were statistically analyzed. The Ki67 levels of the primary and recurrent lesions from 14 OMM patients were compared.

Results: Univariate analysis showed that tumor type and cervical lymph node (CLN) status, as well as Ki67 expression, were all correlated with survival. Cox proportional hazards regression analysis identified Ki67 expression and CLN status as independent prognostic factors in OMM patients. Further, we found that Ki67 expression was associated with clinical tumor type of OMM. Moreover, with a cut-off point of $20 \%$, patients with lower Ki67 scores showed a survival advantage over those with higher Ki67 scores.

Conclusions: Ki67 expression may be a useful pathological predictor of survival of OMM patients without distant metastases.
\end{abstract}

Key words: Oral mucosal melanomas, Ki67 expression, proliferation markers, prognosis, clinical characteristics.

\section{Introduction}

Primary oral mucosal melanomas (OMMs), a set of exceedingly dismal neoplasms, are estimated to comprise $1 \%-2 \%$ of all oral malignancies and account for less than $1 \%$ of all melanomas in the USA and about $7.5 \%$ in Asians [1, 2]. The Western Society of Teachers of Oral Pathology reported that OMMs differed from cutaneous melanomas in histology and that the prognosis of OMMs was poorer than cutaneous melanomas [3]. However, due to their rarity, OMM remains poorly understood, calling for further investigation of its prognostic parameters.
In previous studies, some clinical and pathological features including age at diagnosis, gender, Breslow tumor thickness, and presence of lymph node metastases were suggested as important factors influencing the prognosis of cutaneous melanoma [4]. These indices are not fully fit for OMM, although the understanding of OMM prognosis is still limited. Our previous studies have revealed some clinical features that may be associated with the prognosis of OMM patients, such as tumor type and cervical lymph node (CLN) status [5]. But the 
predictive pathological prognostic factors in OMM remained obscure.

Over-proliferation is a key feature of tumor progression and the proliferation rate is related to prognosis [6]. The tumor proliferation rate is widely estimated immunohistochemically using the Ki67 antibody MIB-1 [7], which identifies the cells of late G1, S, G2, and M phases [8]. The level of Ki67 expression has been used as a prognostic determination index in a number of human cancers [9-12], especially in breast carcinoma [13-15] and cervical cancer [16]. In melanoma, Ki67 is commonly used as an auxiliary index to distinguish melanomas from benign nevi [17], but there is limited information about the relationship of the proliferation rate and prognosis in melanoma. Recently, some studies aimed to clarify whether Ki67 could act as a prognostic index in some subtypes of melanoma [18-21]. However, to the best of our knowledge, it remains unclear whether Ki67 expression could act as a predictive prognostic factor in OMM; furthermore, the correlation of Ki67 expression and clinical features has also not been previously revealed.

Thus, the aims of the present study were to 1) investigate the relationship between the level of Ki67 expression and OS of OMM patients without distal metastasis in order to provide predictive prognostic information of OMM; 2) identify the correlation between Ki67 expression level and tumor characteristics of OMM.

\section{Patients and Methods}

\section{Patients}

This study was approved by the Ethics Committee of the Ninth People's Hospital affiliated to Shanghai JiaoTong University School of Medicine. The clinical manifestations, histopathology, treatment, and outcomes of OMM patients admitted to our hospital from Jan. 2010 to Mar. 2012 were retrospectively analyzed. Patients with the following characteristics were eligible for the study: (1) the primary site of the lesion was the oral mucosa; (2) primary OMMs were histologically diagnosed by biopsy or from surgical specimens by two experienced pathologists; (3) complete clinical, histological and follow-up records were available; and (4) age $\leq 85$ at diagnosis. Patients with (1) an Eastern Cooperative Oncology Group (ECOG) performance status score $\geq 2$ [22]; (2) melanoma at other sites or other malignancies; (3) distant metastasis determined at diagnosis; (4) prior treatment by immunotherapy, chemotherapy or radiotherapy before diagnosis; and (5) who refused to receive treatment were excluded. Finally, a total of 123 patients were included in this study.

Once the diagnosis was confirmed, a radical resection was performed for the primary lesions. Neck dissection was performed for patients in which cervical lymph node metastasis was diagnosed by physical exam combined with ultrasound and/or CT scan. Postoperative chemotherapy with dacarbazine injection (DTIC; Nanjing Pharmaceutical Factory Co. Ltd, Jiangsu, China) and cisplatin injection (CDDP; Qilu Pharmaceutical Co. Ltd, Shandong, China) was repeated every 3 weeks for two cycles for CLN-negative patients and four cycles for CLN-positive patients. DTIC was administered daily on days 2-5 at a dose of $250 \mathrm{mg} / \mathrm{m}^{2}$ and CDDP on day 1 at a dose of $75 \mathrm{mg} / \mathrm{m}^{2}$ (with hydration). If any local recurrences were observed, a repeated radical resection would be considered.

\section{Histology and immunohistochemistry}

The primary disease was diagnosed by biopsy and histology including hematoxylin-eosin staining and immunohistochemical staining of HMB-45, Melan-A, and S-100 protein. Pathological sections from biopsy or radical resection were retrieved for each of the $123 \mathrm{OMM}$ patients. Meanwhile, the primary lesion and recurrence lesion from 14 patients who accepted repeated radical resection were comparatively analyzed. Immunohistochemical staining of Ki67, a widely-used proliferation marker $[7,15,23]$, was performed on the primary lesion as described previously. Sections were de-waxed and rehydrated, and $3 \% \mathrm{H}_{2} \mathrm{O}_{2}$ was used to bleach melanin if necessary and block the activity of endogenous peroxidase. Antigen retrieval was performed by heat treatment for $15 \mathrm{~min}$. Antibodies against Ki67 (1: 200, Santa Cruz Biotechnology, Santa Cruz, CA, USA) were added and incubated overnight at $4^{\circ} \mathrm{C}$. The Dako Real Envision Detection System and AEC peroxidase substrate (Dako, Glostrup, Denmark) were used to detect the primary antibody according to the manufacturer's instructions. To evaluate nonspecific binding, the primary antibody was substituted with PBS.

\section{Evaluation of Ki67 expression}

The pathological parameters were evaluated by two investigators, who were blinded to the patients' clinical data. If their opinions differed, agreement was reached by careful discussion. This evaluation was decisive for the final score. We modified the scoring system slightly as follows: 0 for $<5 \%$ positive cells, 1 for $5 \%-10 \%$ positive cells, 2 for $10 \%-20 \%$ positive cells, 3 for $20 \%-30 \%$ positive cells, 4 for $30 \%-50 \%$ positive cells, and 5 for $>50 \%$ positive cells. 


\section{Statistical analysis}

Survival was measured from the date of pathological diagnosis. Outcome was defined by overall survival (OS). Statistical analysis was performed using SPSS 16.0 for Windows (SPSS Inc., Chicago, IL, USA). Fisher's exact test and chi-square tests were used to compare categorical data. The survival rate was calculated by the Kaplan-Meier method. The prognostic variables included gender, age, primary site, ECOG PS score, tumor type, tumor size, CLN, Ki67 score and therapy mode. The statistical significance of differences between survival curves was established by the log-rank test, and multivariate analysis was performed with the Cox proportional hazard model. The Ki67 levels of the primary and recurrent lesions from OMM patients were compared using the Wilcoxon signed rank test. A two-tailed $P$-value of 0.05 was considered statistically significant.

\section{Results}

\section{Patient characteristics}

A total of 123 patients with OMM were enrolled in this study, comprising 73 males (59.3\%) and 50 females $(40.7 \%)$. The mean age of these patients at first diagnosis was 54 years (22-84 years). The primary lesion was located in the hard palate in 47 patients $(38.2 \%$; Fig. 1A, B), maxillary gingiva in 33 patients (26.8\%; Fig. 1C), mandibular gingiva in 16 patients (13.0\%; Fig. 1D), buccal mucosa in 8 patients $(6.5 \%)$, lips in 11 patients $(8.9 \%$; Fig. 1E, F), and tongue in 8 patients $(6.5 \%)$. The log-rank test showed that the primary site did not correlate with prognosis (Table $1)$. One hundred and nine patients were assigned an ECOG PS score of 0 , while 14 patients were evaluated as 1. As expected, PS score did not correlate with prognosis (Table 1). The size of primary lesion was divided into three grades: grade $1:<2 \mathrm{~cm}, 25$ patients $(20.3 \%)$, grade $2: 2-4 \mathrm{~cm}, 63$ patients $(51.2 \%)$, grade 3 : $>4 \mathrm{~cm}, 35$ patients $(28.5 \%)$. The log-rank test showed that tumor size did not correlate with prognosis. Recurrent lesions were detected in the oral cavity of 36 patients. The range of the follow-up for survivors was $10-78$ months, and the 5 -year OS was $27.5 \%$ (Fig. 2A).

\section{Tumor type and positive cervical lymph node were prognostic factors for OMM}

The tumor type of OMM could be divided clinically into macular type or nodular type (Fig. 1) [5]. The macular type of OMM lesion always has a smooth surface and appears flat with an overlying mucosa. The color of a macular OMM is homogeneous or heterogeneous dark brown and/or black and the border shows a geographic-like morphology. The macular lesion has a texture similar to the surrounding tissue, and it does not bleed easily (Fig. 1A, C, E). Nodular melanoma is usually dark or dull-red in color, forming a raised and irregular nodule with or without pedicle, with a soft texture, and bleeds easily (Fig. 1B, D, F). In the present study, 53 OMMs (43.1\%) were detected with macular lesions and $56.9 \%$ of patients $(\mathrm{n}=70)$ had nodular melanomas. Next, we analyzed the difference in OS between patients with macular and nodular melanoma, and found that patients with nodular melanoma displayed a poorer prognosis compared with patients with macular melanoma (Log-rank = 19.271, $P<0.001$; Fig. 2B), indicating that tumor type may act as a prognostic factor in OMM. Further multivariate analysis suggested that tumor type could not act as an independent prognostic factor for OMM (Table 1).

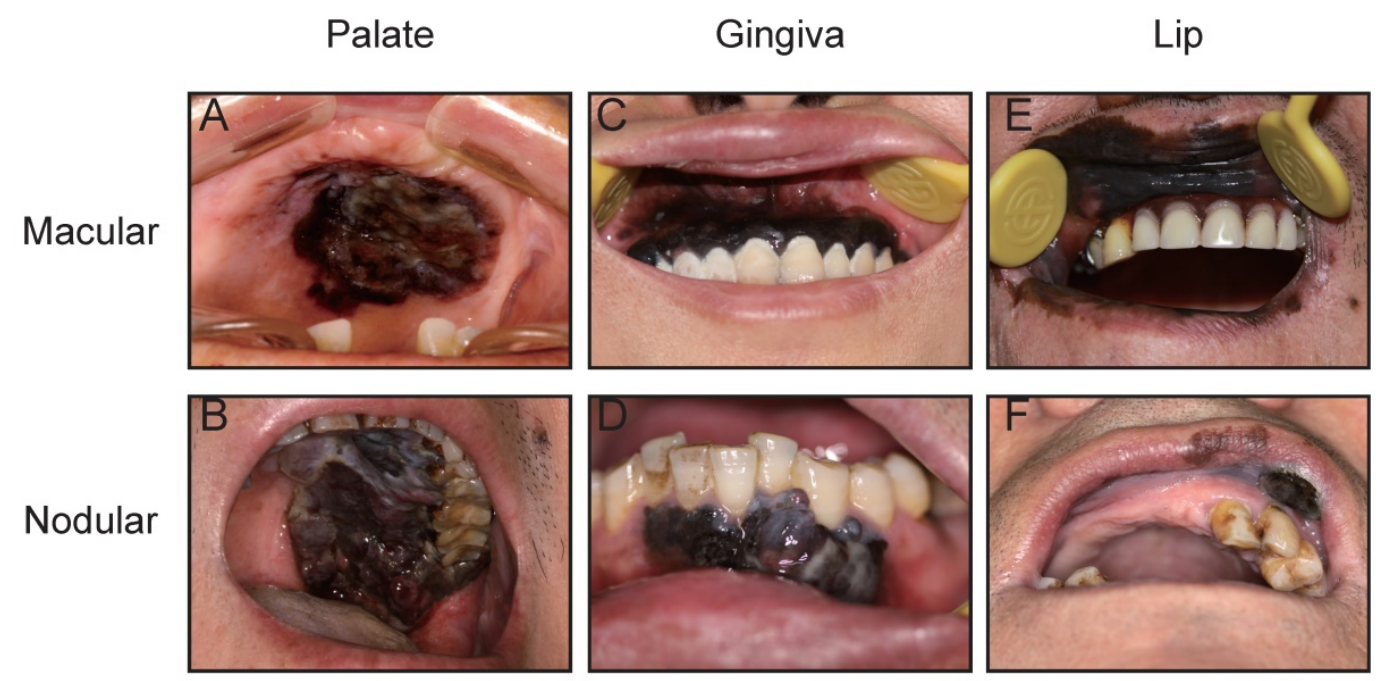

Figure 1. Representative views of OMMs in the hard palate (A, B), gingiva (C, D) and lip (E, F). Macular type (A, C, E) and nodular type (B, D, F) OMMs in the different regions. 
Table 1. The OS of OMM patients by different prognostic variables.

\begin{tabular}{|c|c|c|c|c|c|}
\hline \multirow[t]{2}{*}{ Variable } & \multirow[t]{2}{*}{ No. } & \multirow[t]{2}{*}{$\%$} & \multirow{2}{*}{$\begin{array}{l}\text { Median OS } \\
\text { (months) }\end{array}$} & \multicolumn{2}{|l|}{$P$} \\
\hline & & & & Unviariate & Multivariate \\
\hline \multicolumn{6}{|c|}{ (2) } \\
\hline Male & 73 & 59.3 & 49 & 0.289 & - \\
\hline Female & 50 & 40.7 & 48 & & \\
\hline \multicolumn{6}{|l|}{ Age,years } \\
\hline$<55$ & 54 & 43.9 & 52 & 0.177 & - \\
\hline$\geq 55$ & 69 & 56.1 & 47 & & \\
\hline \multicolumn{6}{|l|}{ Primary site } \\
\hline Palate & 47 & 38.2 & 45 & 0.141 & - \\
\hline Maxillary gingiva & 33 & 26.8 & 52 & & \\
\hline Mandible gingiva & 16 & 13.0 & 42 & & \\
\hline Buccal mucosa & 8 & 6.5 & 46 & & \\
\hline Lips & 11 & 8.9 & 58 & & \\
\hline Tongue & 8 & 6.5 & 55 & & \\
\hline \multicolumn{6}{|l|}{ ECOG PS score } \\
\hline 0 & 109 & 88.6 & 48 & 0.206 & - \\
\hline 1 & 14 & 11.4 & 48 & & \\
\hline \multicolumn{6}{|l|}{ Tumor type } \\
\hline Macular & 53 & 43.1 & 55 & $<0.001$ & 0.165 \\
\hline Nodular & 70 & 56.9 & 43 & & \\
\hline \multicolumn{6}{|l|}{ Tumor size } \\
\hline$\leq 2 \mathrm{~cm}$ & 25 & 20.3 & 55 & 0.257 & - \\
\hline $2-4 \mathrm{~cm}$ & 63 & 51.2 & 47 & & \\
\hline$\geq 4 \mathrm{~cm}$ & 35 & 28.5 & 45 & & \\
\hline \multicolumn{6}{|l|}{ CLN } \\
\hline Positive & 38 & 30.9 & 40 & 0.001 & 0.009 \\
\hline Negative & 85 & 69.1 & 52 & & \\
\hline \multicolumn{6}{|l|}{ Ki67 score } \\
\hline 0 & 27 & 22.0 & 59 & $<0.001$ & $<0.001$ \\
\hline 1 & 19 & 15.4 & 59 & & \\
\hline 2 & 26 & 20.6 & 50 & & \\
\hline 3 & 14 & 11.4 & 38 & & \\
\hline 4 & 24 & 19.5 & 38 & & \\
\hline 5 & 13 & 10.6 & 37 & & \\
\hline
\end{tabular}

CLN metastasis has been reported to be a prognostic factor in a set of malignant tumors of the head and neck [24]. In the present study, a total of 38 patients $(30.9 \%)$ had CLN metastases at diagnosis as confirmed by neck dissection. Figure 2C shows that patients with positive CLNs had worse outcomes than those with negative CLNs, with median OS of 40 and 52 months, respectively (Log-rank $=10.33, P=0.01$ ). Multivariate analysis confirmed that the presence of positive CLNs was an independent negative prognostic factor for OMM (HR, 1.717; 95\% CI, 1.146-2.572; $P=0.009$; Table 1).

\section{Expression of $\mathrm{Ki} 67$ was associated with OS of patients with OMMs}

The proliferation marker, Ki67, has been widely used for assisting with diagnosis of melanoma [17]. The present study analyzed whether Ki67 expression was related to OS using clinicopathological data and follow-up data of the 123 patients. Kaplan-Meier survival curves showed that cases with high Ki67 expression had significantly poorer OS compared with low Ki67 expression cases $(P \quad<0.001)$. Multivariate analysis using the Cox regression hazard model confirmed that Ki67 expression was an independent prognostic factor for poor OS (HR, 1.474; 95\% CI, 1.268-1.713; $P<0.001$; Table 1) among patients with OMM.

\section{Ki67 expression was associated with clinical tumor type of OMM}

The proliferation rate of tumor cells varies among different subtypes of cancers $[25,26]$. In this study, we hypothesized that proliferation rate may be related to clinical characteristics. In order to verify this hypothesis, the correlation between Ki67 expression and clinical features of OMM were analyzed. The expressions of $\mathrm{Ki} 67$ in different clinical features are shown in Table 2 and Figure 4. The chi-square test and Fisher's exact test indicated that there were more Ki67-positive cells in nodular lesions than in macular lesions $(P<0.001)$. As shown, Ki67 expression was not significantly associated with other characteristics including gender, age, primary site, ECOG PS score, tumor size and CLN status. These data suggest that Ki67 expression is associated with tumor type in OMM.
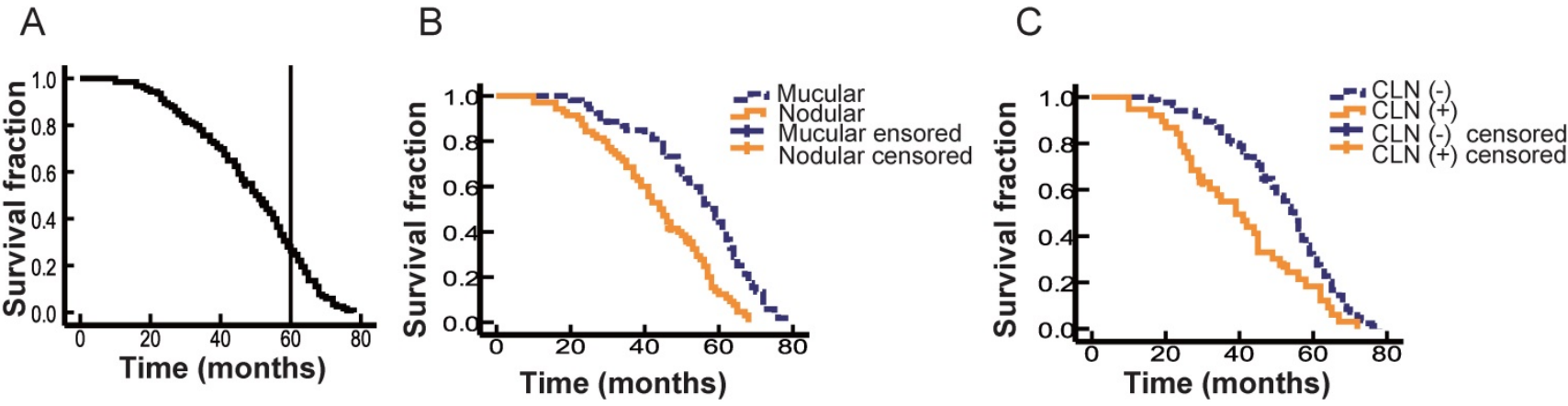

Figure 2. The correlation of OS with different clinical variables. A. Kaplan-Meier curve showing the OS of all 123 cases. B. Kaplan-Meier curves of macular-type lesions (blue, $n=53$ ) and nodular-type lesions (yellow, $n=70)$. C. Kaplan-Meier curves of different cervical lymph node $(C L N)$ status. The blue line indicates patients with negative CLNs $(n=85)$ and the yellow line represents patients with positive CLNs $(n=38)$. 
A

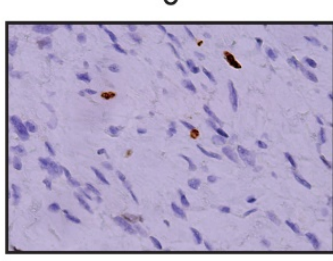

3

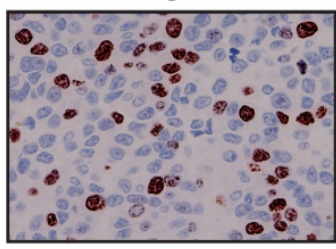

1

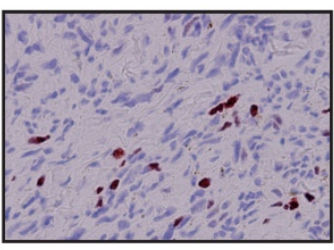

4

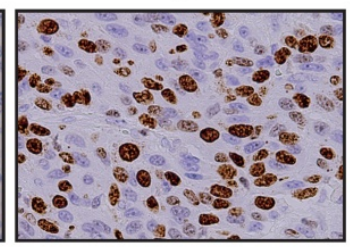

2

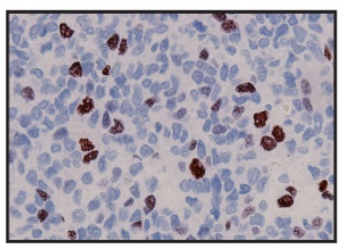

5

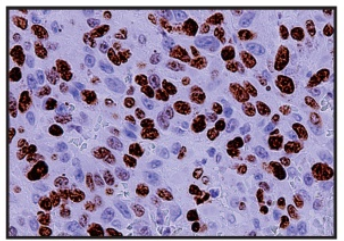

B

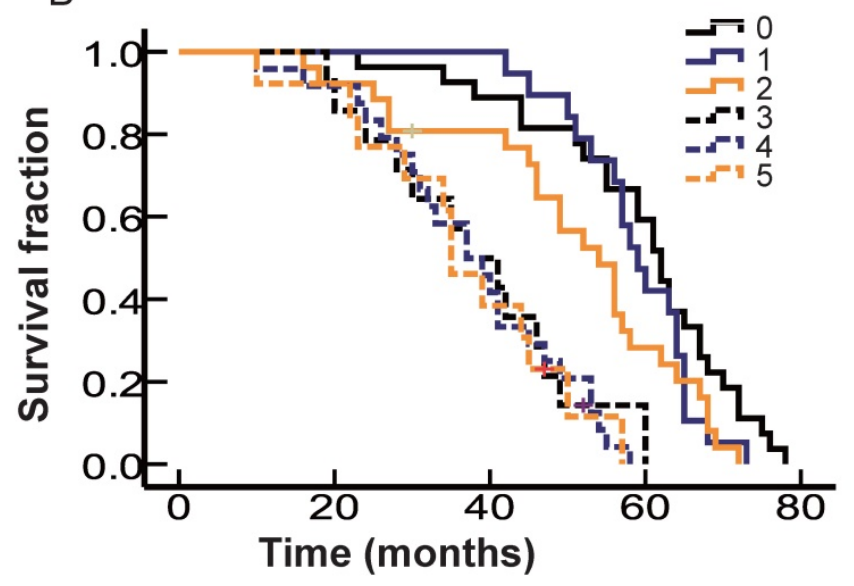

Figure 3. The correlation of OS with differences in the cell proliferation rate in OMM. A. Representative standard of Ki67 score. B. Kaplan-Meier curves of different levels of Ki67 expression. Cases with a Ki67 score of $0-2$ are indicated by a solid blue line $(n=27)$, a solid black line $(n=19)$ and a solid yellow line $(n=26)$. Cases with a Ki67 score of $3-5$ are indicated by a dotted blue line $(n=14)$, dotted black line $(n=24)$ and dotted yellow line $(n=13)$.

A

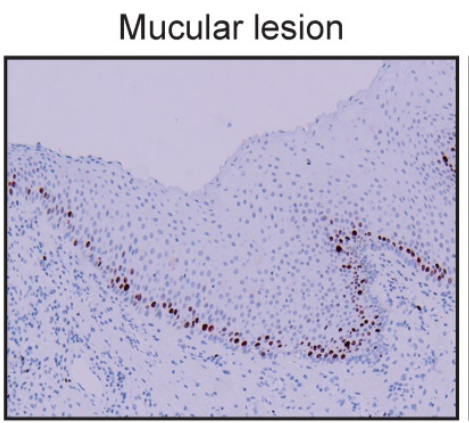

Nodular lesion

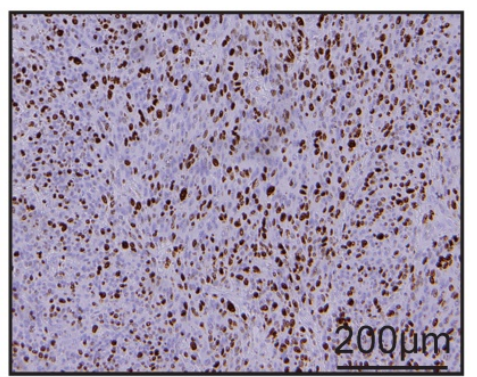

B

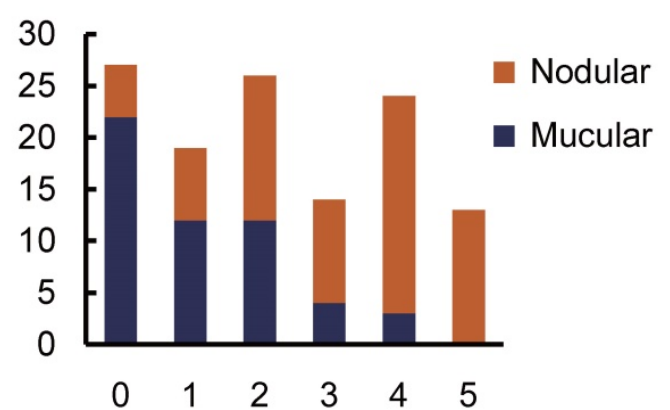

Figure 4. The correlation of tumor type and cell proliferation rate in OMMs. A. Immunohistochemical staining of Ki67 in OMM with macular type lesion and nodular type lesion. B. Bar diagram illustrating the Ki67 score in OMMs of different macular types $(n=53)$ and nodular types $(n=70)$.

\section{Differences in the level of Ki67 expression between primary and recurrent OMMs}

Local recurrence is a difficult problem in the treatment of OMM [27]. Among 123 cases included in the present study, 36 patients suffered from local recurrence. The primary and recurrent paraffin specimens of 14 patients who suffered from local recurrence and underwent repeated radical resection were collected in the present study. We analyze the Ki67 score between primary and recurrent lesions from each case by Wilcoxon signed rank test. As shown in Fig. 5, the expression of Ki67 in recurrent OMM was significantly higher than that in primary OMM $(P=0.03)$. 
Table 2. Correlation of the level of Ki67 expression and different clinical characteristics in OMM.

\begin{tabular}{|c|c|c|c|c|c|c|c|c|c|c|c|c|c|c|c|}
\hline \multirow[t]{2}{*}{ Variable } & \multicolumn{2}{|l|}{0} & \multicolumn{2}{|l|}{1} & \multicolumn{2}{|l|}{2} & \multicolumn{2}{|l|}{3} & \multicolumn{2}{|l|}{4} & \multicolumn{2}{|l|}{5} & \multirow[t]{2}{*}{ Total } & \multicolumn{2}{|c|}{ Ki67 expression } \\
\hline & No. & $\%$ & No. & $\%$ & No. & $\%$ & No. & $\%$ & No. & $\%$ & No. & $\%$ & & $\chi 2$ & $\mathrm{P}$ \\
\hline \multicolumn{16}{|l|}{ Sex } \\
\hline Male & 12 & 16.4 & 10 & 13.7 & 14 & 19.2 & 9 & 12.3 & 19 & 26.0 & 9 & 12.3 & 73 & 7.742 & 0.171 \\
\hline Female & 15 & 30.0 & 9 & 18.0 & 12 & 24.0 & 5 & 10.0 & 5 & 10.0 & 4 & 8.0 & 50 & & \\
\hline \multicolumn{16}{|l|}{ Age,years } \\
\hline$<55$ & 11 & 20.4 & 9 & 16.7 & 9 & 16.7 & 8 & 14.8 & 12 & 22.2 & 5 & 9.3 & 54 & 2.628 & 0.757 \\
\hline$\geq 55$ & 16 & 23.2 & 10 & 14.5 & 17 & 24.6 & 6 & 8.7 & 12 & 17.4 & 8 & 11.6 & 69 & & \\
\hline \multicolumn{16}{|l|}{ Primary site } \\
\hline Palate & 13 & 27.7 & 4 & 8.5 & 11 & 23.4 & 4 & 8.5 & 8 & 17.0 & 7 & 14.9 & 47 & 28.329 & 0.293 \\
\hline Maxillary gingiva & 6 & 18.2 & 8 & 24.2 & 6 & 18.2 & 4 & 12.1 & 8 & 24.2 & 1 & 3.0 & 33 & & \\
\hline Mandible gingiva & 3 & 18.8 & 0 & 0.0 & 3 & 18.8 & 4 & 25.0 & 4 & 25.0 & 2 & 12.5 & 16 & & \\
\hline Buccal mucosa & 1 & 12.5 & 2 & 25.0 & 1 & 12.5 & 1 & 12.5 & 0 & 0.0 & 3 & 37.5 & 8 & & \\
\hline Lips & 2 & 18.2 & 4 & 36.4 & 3 & 27.3 & 0 & 0.0 & 2 & 18.2 & 0 & 0.0 & 11 & & \\
\hline Tongue & 2 & 25.0 & 1 & 12.5 & 2 & 25.0 & 1 & 12.5 & 2 & 25.0 & 0 & 0.0 & 8 & & \\
\hline \multicolumn{16}{|l|}{ ECOG PS score } \\
\hline 0 & 25 & 22.9 & 15 & 13.8 & 23 & 21.1 & 13 & 11.9 & 23 & 21.1 & 10 & 9.2 & 109 & 5.114 & 0.371 \\
\hline 1 & 2 & 14.3 & 4 & 28.6 & 3 & 21.4 & 1 & 7.1 & 1 & 7.1 & 3 & 21.4 & 14 & & \\
\hline \multicolumn{16}{|l|}{ Tumor type } \\
\hline Macular & 22 & 41.5 & 12 & 22.6 & 12 & 22.6 & 4 & 7.5 & 3 & 5.7 & 0 & 0.0 & 53 & 39.653 & 0.000 \\
\hline Nodular & 5 & 7.1 & 7 & 10.0 & 14 & 20.0 & 10 & 14.3 & 21 & 30.0 & 13 & 18.6 & 70 & & \\
\hline \multicolumn{16}{|l|}{ Tumor size } \\
\hline$\leq 2 \mathrm{~cm}$ & 9 & 36.0 & 6 & 24.0 & 4 & 16.0 & 2 & 8.0 & 3 & 12.0 & 1 & 4.0 & 25 & 7.995 & 0.637 \\
\hline $2-4 \mathrm{~cm}$ & 11 & 17.5 & 8 & 12.7 & 16 & 25.4 & 8 & 12.7 & 12 & 19.0 & 8 & 12.7 & 63 & & \\
\hline$\geq 4 \mathrm{~cm}$ & 7 & 20.0 & 5 & 14.3 & 6 & 17.1 & 4 & 11.4 & 9 & 25.7 & 4 & 11.4 & 35 & & \\
\hline \multicolumn{16}{|l|}{ CLN } \\
\hline Positive & 5 & 13.2 & 3 & 7.9 & 11 & 28.9 & 5 & 13.2 & 9 & 23.7 & 5 & 13.2 & 38 & 6.545 & 0.257 \\
\hline Negative & 22 & 25.9 & 16 & 18.8 & 15 & 17.6 & 9 & 10.6 & 15 & 17.6 & 8 & 9.4 & 85 & & \\
\hline
\end{tabular}

A

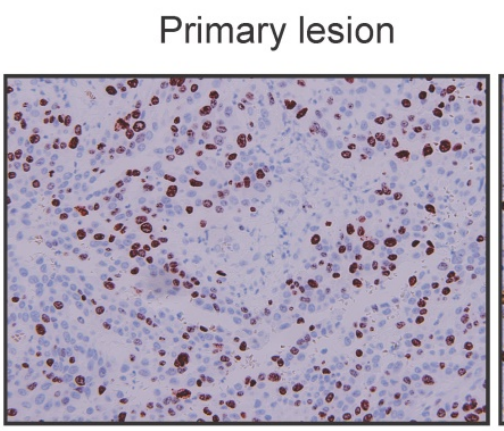

Recurrent lesion

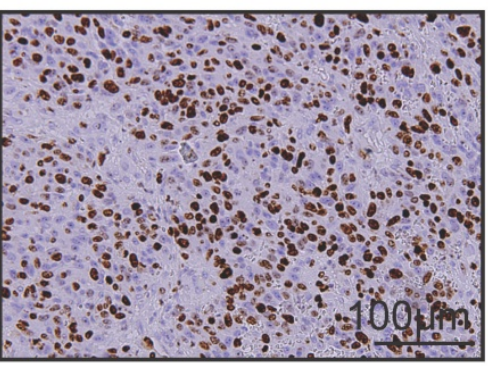

B

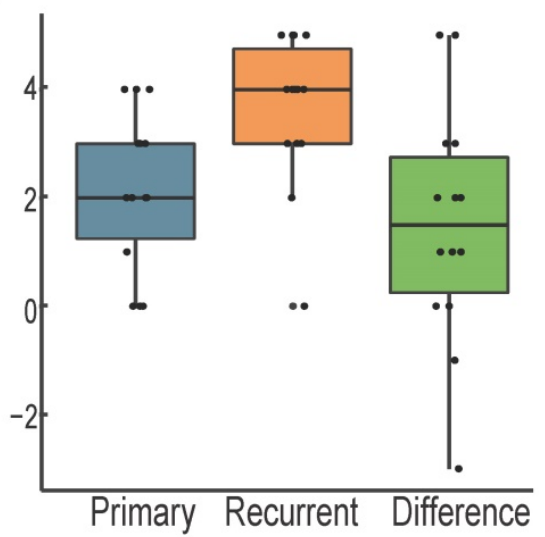

Figure 5. Different levels of Ki67 expression in primary and recurrent OMMs A. Immunohistochemical staining of Ki67 in the primary lesion and the recurrent lesion from the same case of OMM. B. Box diagram illustrating the Ki67 score of primary lesions, recurrent lesions and the difference of the two values.

\section{High expression of $\mathrm{Ki} 67$ was an adverse clinicopathological prognostic marker of OMM}

To further elucidate the relation between Ki67 expression and prognosis of OMM patients, we calculated the cut-off point of the Ki67 expression score by a series of univariate analyses. The results showed that OMM patients with higher Ki67 scores (3-5) showed poor survival time in comparison to those with lower Ki67 scores (0-2) (Log-rank $=57.867$, $P<0.001$, Fig. 6 A).

The present results revealed that CLN status and
Ki67 expression score were independent prognostic factors of OMM (Table 1). Therefore, we analyzed the association between Ki67 expression and OS among patients with different CLN status. Patients with higher Ki67 expression score (3-5) showed significantly poorer OS compared to those with lower score $(0-2)$ in both CLN-negative $(($ Log-rank $=47.635$, $P<0.001$, Fig. 6 B) and CLN-positive groups $(($ Log-rank $=11.561, P=0.001$, Fig. 6 C). All these results indicated that high expression of Ki67 (score above 3) could act as an adverse prognostic factor of OMM. 

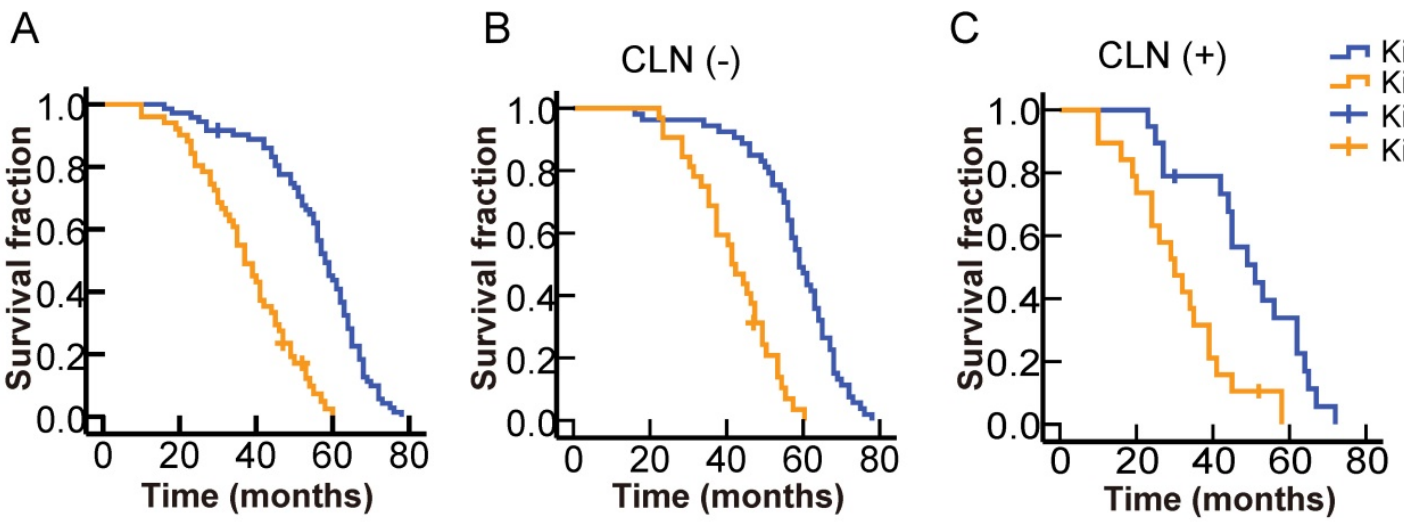

Figure 6. Survival analysis of OMMs with different levels of Ki67 expression and different cervical lymph node (CLN) status. A. Significantly better survival for patients with lower Ki67 scores (0-2) among the total number of cases. B. Significantly better survival for patients with lower Ki67 scores $(0-2)$ among cases without CLN metastasis. C. Significantly better survival for patients with lower Ki67 scores (0-2) among cases with CLN metastasis.

\section{Discussion}

OMM is an extremely rare high-grade malignant tumor with a dismal prognosis [28, 29]. Known for its poorer prognosis compared to cutaneous melanoma, $\mathrm{OMM}$, as a heterogeneous tumor, differs from cutaneous melanoma in several physical, biological, and genetic characteristics $[3,30]$. In addition, due to its rarity, OMM is poorly understood in comparison with other types of melanoma, such as cutaneous melanoma. In this study, we retrospectively analyzed the clinical manifestations, histopathology and outcomes of 123 primary OMM patients without distant metastases hospitalized in a single institution form Jan. 2010 to Mar. 2012. The present study, to the best of our knowledge, includes the biggest study population of OMM without distant metastases which focused on both clinical and histopathological characteristics.

In general, the prognosis of malignancies was closely related to TNM classification. OMM has an extremely poor prognosis following diagnosis of distal metastasis (life expectancy is only 3-8 months) $[5,31,32]$, cases with distal metastasis were excluded from this study. Clinical characteristics including gender, age, primary site, tumor type, tumor size and CLN status of patients with OMM were analyzed in the remaining 123 patients.

Macular OMM and nodular OMM are the most common types observed in the clinic [5,33]. In our previous study, we found that the nodular type is a dangerous signal in OMM [5]. In this study, 53 OMMs $(43.1 \%)$ were detected with macular melanoma and 70 $(56.9 \%)$ had nodular lesions. Survival analysis indicated that the tumor type could be associated with poor outcome but could not act as an independent prognostic factor for OMM. However, consistent with our previous study, the presence of metastatic CLNs was an independent prognostic factor for OMM [5].
These data indicate that tumor type and CLN status may act as clinical prognostic factors in OMM patients without distal metastasis.

Cell proliferation rate is considered as an important prognostic factor in a series of malignant tumors [15, 34]. The presence of Ki67 protein is an indicator of proliferative activity and is detected in the nucleus of cells during late G1, S, G2, and M phases, thus staining most cells in the cell cycle [8]. A series of studies have indicated that Ki67 could be used as a predictive and prognostic marker in a number of cancers including prostate cancer, gastrointestinal stromal tumors, clear-cell renal cell cancer, epithelial ovarian cancer and non-small-cell lung cancer [9-13, $16,35,36]$. In a meta-analysis of 64,196 breast cancer patients, authors concluded that Ki67 has an independent poor prognostic value in terms of OS [37]. Meanwhile a large case and long-term follow-up study suggested that the use of Ki67 expression as an index for classifying patients with breast cancer into different risk categories [14, 38]. But there is limited information involving Ki67 expression in melanoma. The previous studies, mainly involving cutaneous melanoma, focused on the value of Ki67 expression in the differential diagnosis of benign and malignant tumors [17]. In cutaneous melanoma, the Ki67 expression index is demonstrated to correlate with tumor grade and patient survival $[18,39]$. There were only a few studies with small sample sizes which suggested that Ki67 expression may be related to the prognosis of sinonasal and anorectal mucosal melanoma [20, 21, 34]. However, information about the relation between Ki67 expression and the prognosis of OMM is still lacking. In this study, we provide evidence that cell proliferation rate is associated with the prognosis of OMM by evaluating the expression of Ki67 in the primary tumor of OMM patients without distant metastases. It has been suggested that the level of Ki67 expression could be 
used as a decision-making tool during chemotherapy treatment of breast cancer $[40,41]$. Whether the Ki67 proliferation index could play a similar role in OMM needs to be investigated in future research.

The American Joint Committee on Cancer released the staging system for head and neck mucosal melanoma in 2010 [42], but the histopathological classification and microstaging of OMM has not been established. In mucosal melanoma, tumor thickness is an important histological parameter of prognostic significance. However, due to the special anatomic characteristics of OMM, it is hard to judge its exact thickness. Thus, markers for risk assessment in individual patients are still needed. In the present study, we analyzed the relation between the expression of Ki67 in the primary tumors and survival time of 123 OMM patients. Our results identified that Ki67 expression was an adverse prognostic marker for OS time among patients with OMM and could act as an independent prognostic factor for OMMs, which may be developed as a useful histological parameter of prognostic significance of OMM without distant metastases in the future. In a study of 30 patients with anorectal malignant melanoma, the OS of 20 patients with higher Ki67 index scores was significantly poorer than that of 10 who had lower Ki67 index scores [20]. A study on sinonasal mucosal melanoma also found a significant difference among different Ki67 index scores [21]. Whereas, a research with seven primary esophageal malignant melanoma patients and three primary anorectal malignant melanoma patients found no correlation between Ki67 expression and prognosis [43]. The negative result may be due to the small sample size and different lesion locations.

The association between the Ki67 expression and clinical characteristics were noticed in this study. Tumor type, unlike other characteristics such as gender, age, primary site, tumor size and CLN status, was discovered to be related to the Ki67 expression level. Thus, cases with nodular lesions are more likely to have a higher Ki67 expression. In previous studies of breast cancer, similar phenomena have been observed: the Ki67 index could act as an important parameter to distinguish specific subtypes of breast cancer [44, 45]. A study in cutaneous melanoma indicated that there was a relation between Ki67 protein expression and clinical features. Researchers observed tumors with overexpression of Ki67 (more than 10\%) were thicker than those with low Ki67 expression [18]. Combined with the data showing that nodular tumors show a significantly poorer OS than macular melanoma in univariate analysis, while it could not act as an independent prognostic factor in multivariate analysis when adjusted by Ki67 expression, Ki67 expression was associated with clinical tumor type. We suspect that the proliferation rate of tumor cells forms the underpinning of significant differences in clinical outcome observed between tumor types, although further data are needed in the future to confirm this.

Local recurrence is a common problem in OMM. The high rate of local recurrence may result from the complicated anatomic, non-apparent diffusion of tumor cells via the submucosal lymphatic route or the presence of multifocal lesions [46]. The local recurrence rate was $29.3 \%$ in this study, which is similar to that observed in previous studies [27]. The expression level of Ki67 was analyzed in 14 cases with primary and recurrent lesions and remarkable differences were observed. Similar characteristics of Ki67 expression were seen in a number of other cancers [47-49]. It was first observed in OMM in our study, though with a small sample, and more studies are needed to show the relation between the proliferation rate and local recurrence of OMM.

The results from the present study demonstrate that the expression of Ki67 is closely related to clinical prognosis of OMM and can be considered to be an independent predictor of prognosis in OMM patients. To improve the feasibility of clinical application, we attempted to discover a convenient and efficient parameter of Ki67 expression score. Our results indicated that a Ki67 expression score of 3 (Ki67 cell positivity rate of more than $20 \%$ ) was the most efficient cut-off point for discriminating the survival curves of distant metastases-free OMM, and was verified in different CLN status which was demonstrated to be the other independent prognostic factor in this study. In a study of anorectal malignant melanoma the cut-off point was $40 \%$, while in a study of sinonasal mucosal melanoma assigned a cut-off value of $35 \%[20,21]$. The disparity in the cut-off points in the studies is possibly because of the difference in the mean expression of the Ki67 marker in the different locations of melanomas [50]. Thus, the clinicopathological parameter, Ki67 expression level, is expected to be a possible candidate reference for prognostic evaluation of OMM.

In summary, the present study demonstrates that Ki67 could act as an important molecular biological indicator for predicting the prognosis of distant metastases-free OMM patients. Further, Ki67 is a potential candidate for pathological grading and staging of OMM in the future.

\section{Abbreviations}

OMM: oral mucosal melanoma; OS: overall survival; CLN: cervical lymph node; ECOG: Eastern Cooperative Oncology Group; 


\section{Acknowledgements}

The authors thank Jiang $\mathrm{Li}$ and Ting $\mathrm{Gu}$ (Department of Oral Pathology, Ninth People's Hospital, School of Medicine, Shanghai JiaoTong University, China) for technical support.

This work was supported by the Project of Science and Technology Commission of Shanghai Municipality (grant numbers: 10410711200, 08140902100, 11495802000, 14DZ1941400 and 14DZ1941402) The National Key Research and Development Program (grant number: 2016YFC0905000 and 2016YFC0905003) and Shanghai Health and Family Planning Commission (No.20154Y0057).

\section{Competing Interests}

The authors have declared that no competing interest exists.

\section{References}

1. Chang AE, Karnell LH, Menck HR. The National Cancer Data Base report on cutaneous and noncutaneous melanoma: a summary of 84,836 cases from the past decade. The American College of Surgeons Commission on Cancer and the American Cancer Society. Cancer. 1998; 83: 1664-78.

2. Umeda M, Shimada K. Primary malignant melanoma of the oral cavity--its histological classification and treatment. The British journal of oral \& maxillofacial surgery. 1994; 32: 39-47.

3. Barker BF, Carpenter WM, Daniels TE, Kahn MA, Leider AS, Lozada-Nur F, et al. Oral mucosal melanomas: the WESTOP Banff workshop proceedings. Western Society of Teachers of Oral Pathology. Oral surgery, oral medicine, oral pathology, oral radiology, and endodontics. 1997; 83: 672-9.

4. Jiang Y, Shi X, Zhao Q, Krauthammer M, Rothberg BE, Ma S. Integrated analysis of multidimensional omics data on cutaneous melanoma prognosis. Genomics. 2016; 107: 223-30.

5. Wu Y, Zhong Y, Li C, Song H, Guo W, Ren G. Neck dissection for oral mucosal melanoma: caution of nodular lesion. Oral oncology. 2014; 50: 319-24.

6. Li CF, Chen LT, Lan J, Chou FF, Lin CY, Chen YY, et al. AMACR amplification and overexpression in primary imatinib-naive gastrointestinal stromal tumors: a driver of cell proliferation indicating adverse prognosis. Oncotarget. 2014; 5: 11588-603.

7. Gerdes J, Schwab U, Lemke H, Stein H. Production of a mouse monoclonal antibody reactive with a human nuclear antigen associated with cell proliferation. International journal of cancer. 1983; 31: 13-20.

8. van Diest PJ, Brugal G, Baak JP. Proliferation markers in tumours: interpretation and clinical value. Journal of clinical pathology. 1998; 51: 716-24.

9. Basilio-de-Oliveira RP, Pannain VL. Prognostic angiogenic markers (endoglin, VEGF, CD31) and tumor cell proliferation (Ki67) for gastrointestinal stromal tumors. World journal of gastroenterology. 2015; 21: 6924-30.

10. Gayed BA, Youssef RF, Bagrodia A, Darwish OM, Kapur P, Sagalowsky A, et al. Ki67 is an independent predictor of oncological outcomes in patients with localized clear-cell renal cell carcinoma. BJU International. 2014; 113: 668-73.

11. Pillai K, Pourgholami MH, Chua TC, Morris DL. Prognostic significance of Ki67 expression in malignant peritoneal mesothelioma. American journal of clinical oncology. 2015; 38: 388-94.

12. Pascale M, Aversa C, Barbazza R, Marongiu B, Siracusano S, Stoffel F, et al. The proliferation marker Ki67, but not neuroendocrine expression, is an independent factor in the prediction of prognosis of primary prostate cancer patients. Radiology and oncology. 2016; 50: 313-20.

13. Dowsett M, Nielsen TO, A'Hern R, Bartlett J, Coombes RC, Cuzick J, et al. Assessment of Ki67 in breast cancer: recommendations from the International Ki67 in Breast Cancer working group. Journal of the National Cancer Institute. 2011; 103: 1656-64

14. Bustreo S, Osella-Abate S, Cassoni P, Donadio M, Airoldi M, Pedani F, et al. Optimal Ki67 cut-off for luminal breast cancer prognostic evaluation: a large case series study with a long-term follow-up. Breast cancer research and treatment. 2016; 157: 363-71.

15. Pathmanathan N, Balleine RL. Ki67 and proliferation in breast cancer. Journal of clinical pathology. 2013; 66: 512-6

16. Ikenberg H, Bergeron C, Schmidt D, Griesser H, Alameda F, Angeloni C, et al. Screening for cervical cancer precursors with p16/Ki-67 dual-stained cytology: results of the PALMS study. Journal of the National Cancer Institute. 2013; 105: 1550-7.
17. Wandler A, Spaun E, Steiniche T, Nielsen PS. Automated quantification of Ki67/MART1 stains may prevent false-negative melanoma diagnoses. Journal of cutaneous pathology. 2016; 43: 956-62.

18. Vaisanen A, Kuvaja P, Kallioinen M, Turpeenniemi-Hujanen T. A prognostic index in skin melanoma through the combination of matrix metalloproteinase-2, Ki67, and p53. Human pathology. 2011; 42: 1103-11.

19. Nielsen PS, Riber-Hansen R, Jensen TO, Schmidt H, Steiniche T. Proliferation indices of phosphohistone $\mathrm{H} 3$ and Ki67: strong prognostic markers in a consecutive cohort with stage I/II melanoma. Modern pathology : an official journal of the United States and Canadian Academy of Pathology, Inc. 2013; 26: 404-13

20. Ben-Izhak O, Bar-Chana M, Sussman L, Dobiner V, Sandbank J, Cagnano M, et al. Ki67 antigen and PCNA proliferation markers predict survival in anorectal malignant melanoma. Histopathology. 2002; 41: 519-25.

21. Kim DK, Kim DW, Kim SW, Kim DY, Lee CH, Rhee CS. Ki67 antigen as a predictive factor for prognosis of sinonasal mucosal melanoma. Clinical and experimental otorhinolaryngology. 2008; 1: 206-10.

22. Oken MM, Creech RH, Tormey DC, Horton J, Davis TE, McFadden ET, et al. Toxicity and response criteria of the Eastern Cooperative Oncology Group. American journal of clinical oncology. 1982; 5: 649-55.

23. Urruticoechea A, Smith IE, Dowsett M. Proliferation marker Ki-67 in early breast cancer. Journal of clinical oncology : official journal of the American Society of Clinical Oncology. 2005; 23: 7212-20.

24. Lydiatt WM, Patel SG, O'Sullivan B, Brandwein MS, Ridge JA, Migliacci JC, et al. Head and Neck cancers-major changes in the American Joint Committee on cancer eighth edition cancer staging manual. CA: a cancer journal for clinicians. 2017; 67: 122-37.

25. Kobel M, Kalloger SE, Boyd N, McKinney S, Mehl E, Palmer C, et al. Ovarian carcinoma subtypes are different diseases: implications for biomarker studies. PLoS medicine. 2008; 5: e232.

26. Horlock NM, Wilson GD, Daley FM, Richman PI, Sanders R. Cellular proliferation characteristics of basal cell carcinoma: relationship to clinical subtype and histopathology. European journal of surgical oncology : the journal of the European Society of Surgical Oncology and the British Association of Surgical Oncology. 1997; 23: 247-52.

27. Bakkal FK, Basman A, Kizil Y, Ekinci O, Gumusok M, Ekrem Zorlu M, et al. Mucosal melanoma of the head and neck: recurrence characteristics and survival outcomes. Oral surgery, oral medicine, oral pathology and oral radiology. 2015; 120: 575-80.

28. Meleti M, Leemans CR, Mooi WJ, Vescovi P, van der Waal I. Oral malignant melanoma: a review of the literature. Oral oncology. 2007; 43: 116-21.

29. Mihajlovic M, Vlajkovic S, Jovanovic P, Stefanovic V. Primary mucosal melanomas: a comprehensive review. International journal of clinical and experimental pathology. 2012; 5: 739-53.

30. Postow MA, Hamid O, Carvajal RD. Mucosal melanoma: pathogenesis, clinical behavior, and management. Current oncology reports. 2012; 14: 441-8.

31. Balch CM, Gershenwald JE, Soong SJ, Thompson JF, Atkins MB, Byrd DR, et al. Final version of 2009 AJCC melanoma staging and classification. Journal of clinical oncology : official journal of the American Society of Clinical Oncology. 2009; 27: 6199-206

32. Agarwala SS. Current systemic therapy for metastatic melanoma. Expert review of anticancer therapy. 2009; 9: 587-95.

33. Tanaka N, Mimura M, Kimijima Y, Amagasa T. Clinical investigation of amelanotic malignant melanoma in the oral region. Journal of oral and maxillofacial surgery : official journal of the American Association of Oral and Maxillofacial Surgeons. 2004; 62: 933-7.

34. Li LT, Jiang G, Chen Q, Zheng JN. Ki67 is a promising molecular target in the diagnosis of cancer (review). Molecular medicine reports. 2015; 11: 1566-72.

35. Adams SF, Levine DA, Cadungog MG, Hammond R, Facciabene A, Olvera N, et al. Intraepithelial $\mathrm{T}$ cells and tumor proliferation: impact on the benefit from surgical cytoreduction in advanced serous ovarian cancer. Cancer. 2009; 115: 2891-902.

36. Lei B, Liu S, Qi W, Zhao Y, Li Y, Lin N, et al. PBK/TOPK expression in non-small-cell lung cancer: its correlation and prognostic significance with Ki67 and p53 expression. Histopathology. 2013; 63: 696-703.

37. Petrelli F, Viale G, Cabiddu M, Barni S. Prognostic value of different cut-off levels of Ki-67 in breast cancer: a systematic review and meta-analysis of 64,196 patients. Breast cancer research and treatment. 2015; 153: 477-91.

38. Aleskandarany MA, Rakha EA, Macmillan RD, Powe DG, Ellis IO, Green AR. MIB1/Ki-67 labelling index can classify grade 2 breast cancer into two clinically distinct subgroups. Breast cancer research and treatment. 2011; 127: 591-9.

39. Vereecken P, Laporte M, Heenen M. Significance of cell kinetic parameters in the prognosis of malignant melanoma: a review. Journal of cutaneous pathology. 2007; 34: 139-45.

40. Yoshioka T, Hosoda M, Yamamoto M, Taguchi K, Hatanaka KC, Takakuwa E, et al. Prognostic significance of pathologic complete response and Ki67 expression after neoadjuvant chemotherapy in breast cancer. Breast Cancer. 2015; 22: 185-91.

41. Sueta A, Yamamoto $\mathrm{Y}$, Hayashi M, Yamamoto S, Inao T, Ibusuki M, et al. Clinical significance of pretherapeutic $\mathrm{Ki} 67$ as a predictive parameter for response to neoadjuvant chemotherapy in breast cancer: is it equally useful across tumor subtypes? Surgery. 2014; 155: 927-35.

42. Egner JR. AJCC Cancer Staging Manual. JAMA. 2010; 304: 1726-7. 
43. Izumi D, Ishimoto T, Yoshida N, Nakamura K, Kosumi K, Tokunaga R, et al. A clinicopathological analysis of primary mucosal malignant melanoma. Surgery today. 2015; 45: 886-91.

44. Cheang MC, Chia SK, Voduc D, Gao D, Leung S, Snider J, et al. Ki67 index, HER2 status, and prognosis of patients with luminal B breast cancer. Journal of the National Cancer Institute. 2009; 101: 736-50.

45. Mori N, Ota H, Mugikura S, Takasawa C, Ishida T, Watanabe G, et al. Luminal-type breast cancer: correlation of apparent diffusion coefficients with the Ki-67 labeling index. Radiology. 2015; 274: 66-73.

46. Patel SG, Prasad ML, Escrig M, Singh B, Shaha AR, Kraus DH, et al. Primary mucosal malignant melanoma of the head and neck. Head \& neck. 2002; 24 : 247-57.

47. Nofech-Mozes S, Ismiil N, Dube V, Saad RS, Khalifa MA, Moshkin O, et al. Immunohistochemical characterization of primary and recurrent adult granulosa cell tumors. International journal of gynecological pathology : official journal of the International Society of Gynecological Pathologists. 2012; 31: 80-90.

48. Yang Y, Xuan J, Yang Z, Han A, Xing L, Yue J, et al. The expression of epidermal growth factor receptor and $\mathrm{Ki} 67$ in primary and relapse nasopharyngeal cancer: a micro-evidence for anti-EGFR targeted maintenance therapy. Med Oncol. 2012; 29: 1448-55.

49. Tang X, Yao Y, Zhu J, Jin K, Wang Y, Mao Y, et al. Differential proliferative index of cancer stem-like cells in primary and recurrent medulloblastoma in human. Child's nervous system : ChNS : official journal of the International Society for Pediatric Neurosurgery. 2012; 28: 1869-77.

50. Alaeddini M, Etemad-Moghadam S. Immunohistochemical profile of oral mucosal and head and neck cutaneous melanoma. Journal of oral pathology \& medicine : official publication of the International Association of Oral Pathologists and the American Academy of Oral Pathology. 2015; 44: 234-8. 